\title{
A Young Cooling Neutron Star in the Remnant of Supernova 1987A
}

\author{
P. S. Shternin and D. G. Yakovlev* \\ Ioffe Physicotechnical Institute, Russian Academy of Sciences, ul. Politekhnicheskaya 26, St. Petersburg, 194021 \\ Russia \\ Received March 18, 2008
}

\begin{abstract}
We describe the cooling theory for isolated neutron stars that are several tens of years old. Their cooling differs greatly from the cooling of older stars that has been well studied in the literature. It is sensitive to the physics of the inner stellar crust and even to the thermal conductivity of the stellar core, which is never important at later cooling stages. The absence of observational evidence for the formation of a neutron star during the explosion of Supernova 1987A is consistent with the fact that the star was actually born there. It may still be hidden in the dense center of the supernova remnant. If, however, the star is not hidden, then it should have a low thermal luminosity (below $\sim 10^{34} \mathrm{erg} \mathrm{s}^{-1}$ ) and a short internal thermal relaxation time (shorter than $13 \mathrm{yr}$ ). This requires that the star undergo intense neutrino cooling (e.g., via the direct Urca process) and have a thin crust with strong superfluidity of free neutrons and/or an anomalously high thermal conductivity.
\end{abstract}

PACS numbers : 97.58.Mj; $97.60 . \mathrm{Jd}$

DOI: $10.1134 / \mathrm{S} 1063773708100034$

Key words: X-ray sources, Supernova 1987 A, neutron stars.

\section{INTRODUCTION}

The explosion of Supernova (SN) 1987A was of immense importance in understanding the many processes that accompany gravitational collapse and shell expansion (see, e.g., Imshennik and Nadyozhin 1988; Arnett 1996; Immler et al. 2007). The confident identification of the progenitor star (the blue supergiant Sk 1) and the detection of a neutrino burst suggested that gravitational collapse had occurred. The collapse of such a star (with a mass $\sim 20 M_{\odot}$ ) is currently believed to produce a compact object, most likely a neutron star. However, numerous attempts to detect the newly born star (by various methods and in various spectral ranges) have failed. We will analyze how much the nondetection of a neutron star over more than 20 years of observations is compatible with the neutron star cooling theory.

\section{SEARCHING FOR THE NEUTRON STAR}

The young neutron star in the remnant of SN 1987A should be an intense source of soft $X$ rays. As will be shown below, the effective surface temperature of a cooling isolated neutron star with an age $t \sim$ $10-30 \mathrm{yr}$ does not exceed $T_{\mathrm{s}}^{\infty} \lesssim(3-6) \times 10^{6} \mathrm{~K}$. The superscript $\infty$ denotes the temperature recorded by a remote observer. For a blackbody spectrum of

\footnotetext{
*E-mail: yak@astro.ioffe.ru
}

thermal radiation, the intensity reaches its maximum at photon energy $h \nu \lesssim 0.7-1.5 \mathrm{keV}$. The outer layers of the expanding SN 1987A remnant are already transparent in all spectral ranges, but its inner part is still opaque and can hide the neutron star (see, e.g., Fransson and Chevalier 1987; Burrows et al. 2000; Shtykovskiy et al. 2005; Manchester 2007; and references therein). In soft $\mathrm{X}$ rays, the $\mathrm{SN}$ remnant becomes increasingly bright (owing to its expansion), which makes it difficult to detect a point source.

As yet no point $\mathrm{X}$-ray source has been detected in the SN 1987A remnant, but upper limits on its luminosity have been established. Thus, for example, the Chandra observations in 2000 yielded an upper limit on the soft X-ray luminosity $L_{\gamma}^{\infty}(0.5-2 \mathrm{keV})<2.3 \times$ $10^{34} \mathrm{erg} \mathrm{s}^{-1}$ (Burrows et al. 2000). Having processed the Chandra observations for 1999-2002 in harder $\mathrm{X}$ rays, Park et al. (2004) obtained $L_{\gamma}^{\infty}(2-10 \mathrm{keV})<$ $1.5 \times 10^{34} \mathrm{erg} \mathrm{s}^{-1}$. Shtykovskiy et al. (2005) used the XMM-Newton data for 2000 and 2001 to obtain $L_{\gamma}^{\infty}(2-10 \mathrm{keV})<5 \times 10^{34} \mathrm{erg} \mathrm{s}^{-1}$. Based on the XMM-Newton observations in 2000, Haberl et al. (2006) gave $L_{\gamma}^{\infty}(0.2-10 \mathrm{keV})<5.7 \times 10^{34} \mathrm{erg} \mathrm{s}^{-1}$ and $L_{\gamma}^{\infty}(0.5-2 \mathrm{keV})<2.1 \times 10^{34} \mathrm{erg} \mathrm{s}^{-1}$. Finally, Shtykovskiy et al. (2005) processed the INTEGRAL observations for 2003 in the hard X-ray range (20$60 \mathrm{keV})$. Extrapolating the results to the softer X- 
ray range, they obtained the faintest upper limit $L_{\gamma}^{\infty}(2-10 \mathrm{keV})<(0.6-1.6) \times 10^{36} \mathrm{erg} \mathrm{s}^{-1}$.

Given the aforesaid, we assume that the upper limit on the thermal X-ray luminosity of the neutron star (unobscured by the shell) is

$$
L_{\gamma}^{\infty}<2 \times 10^{34} \mathrm{erg} \mathrm{s}^{-1} \text { for } t \approx 13-14 \mathrm{yr} .
$$

If the star is hidden in the dense center of the $\mathrm{SN}$ remnant, then its true luminosity can be even higher. Assuming that $L_{\gamma}^{\infty}=4 \pi \sigma R_{\infty}^{2}\left(T_{\mathrm{s}}^{\infty}\right)^{4}$ and taking a typical "apparent" stellar radius, $R_{\infty}=14 \mathrm{~km}$ (Haensel et al. 2007), we will obtain $T_{\mathrm{s}}^{\infty}<2 \times 10^{6} \mathrm{~K}$ from (1).

Note also the upper limit on the optical and ultraviolet luminosity of the point source in the SN 1987A remnant, $L_{\gamma}^{\infty}(2900-9650 \AA)<(5-8) \times 10^{33} \mathrm{erg} \mathrm{s}^{-1}$ derived by Graves et al. (2005) from the Hubble observations in 1999 and 2003. For a thermal source with an intensity maximum in the soft $\mathrm{X}$-ray range, this limit is fainter than the X-ray limit (1) and is disregarded below.

Finally, we will mention the unsuccessful searches for a pulsating source (pulsar) in various spectral ranges, particularly in the radio and optical ones (see, e.g., Percival et al. 1995; Manchester 2007; and references therein). The absence of a pulsar can be explained by mass accretion from a dense cloud onto the neutron star (see, e.g., Woosley and Weaver 1995). The stellar magnetic field may be buried under the accreted matter; it will take a long time for this field to diffuse outward and for the star to become a pulsar (Muslimov and Page 1995; Geppert et al. 1999).

\section{COOLING MODELS FOR YOUNG NEUTRON STARS}

According to present-day theories, neutron stars are born hot, with an internal temperature of $\sim 10^{11} \mathrm{~K}$. After a short (several tens of seconds) proto-neutron star stage, the formed star becomes transparent to neutrino emission. It cools for $t \sim 10^{4}-10^{5} \mathrm{yr}$ mainly via neutrino emission from its interior and, subsequently, via photon emission from its surface (see, e.g., Yakovlev and Pethick 2004; Page et al. 2006). We are interested in the cooling of a neutron star that is several tens of years old. No thermal radiation has ever been observed from such stars, while only a few papers are devoted to their cooling (Nomoto and Tsuruta 1987; Lattimer et al. 1994; Gnedin et al. $2001)$.

For the purposes of illustration, we will provide the calculations that were performed using our cooling code (Gnedin et al. 2001), which includes the effects of general relativity on the structure and evolution of stars. To simplify the description of our calculations, recall that the outer and inner crusts as well as the outer and inner cores can be distinguished in a neutron star (see, e.g., Haensel et al. 2007). The outer crust extends to a density of $\sim 4 \times 10^{11} \mathrm{~g} \mathrm{~cm}^{-3}$ and is composed of electrons and atomic nuclei. The inner crust extends to a density of $\approx 1.4 \times 10^{14} \mathrm{~g} \mathrm{~cm}^{-3}$ and is composed of electrons, atomic nuclei, and free neutrons. The stellar core is composed of homogeneous asymmetric nuclear matter. The inner core begins at a density of the order of several nuclear densities. The boundary between the outer and inner cores is often not defined well enough. We will assume that this boundary corresponds to the direct Urca threshold (see below).

Let us consider the models of neutron stars whose cores are composed of nucleons, electrons, and muons and have a moderately stiff phenomenological equation of state suggested by Prakash et al. (1988). It corresponds to the compression modulus of equilibrium symmetric nuclear matter $K=180 \mathrm{MeV}$ and the functional form of the symmetry energy suggested by Page and Applegate (1992). The gravitational mass of the most massive stable neutron star with this equation of state is $M_{\max }=1.713 M_{\odot}$; the circumferential radius of this star is $R=9.59 \mathrm{~km}$ and its apparent radius is $R_{\infty}=R / \sqrt{1-r_{g} / R}=13.94 \mathrm{~km}$ ( $r_{g}=2 G M / c^{2}$ is the Schwarzschild radius). The central density of this star is $\rho_{\mathrm{c}}=3.33 \times 10^{15} \mathrm{~g} \mathrm{~cm}^{-3}$. The threshold density for opening a powerful neutrino direct Urca process involving electrons (Lattimer et al. 1991) for the equation of state in question is $\rho_{\text {th }}=1.185 \times 10^{15} \mathrm{~g} \mathrm{~cm}^{-3}$, while the threshold density for the direct Urca process involving muons is slightly higher and does not affect noticeably the cooling (Bejger et al. 2003). The mass of a neutron star with the central density $\rho_{\mathrm{c}}=\rho_{\text {th }}$ is $M_{\text {th }}=1.343 M_{\odot}$ (at $R=11.61 \mathrm{~km}$ and $R_{\infty}=14.30 \mathrm{~km}$ ). In stars with $M<M_{\text {th }}$, the direct Urca process of neutrino cooling is forbidden and they cannot cool too rapidly. In more massive stars, the direct Urca process is open and can greatly accelerate the cooling (unless it is suppressed by nucleon superfluidity). For other equations of state of nucleon matter that permit the direct Urca process, the cooling curves change quantitatively but not qualitatively (Gnedin et al. 2001; Yakovlev et al. 2001).

In several calculations, we will take into account the superfluidity in the stellar core. We will choose a typical model of superfluidity that can explain the observations of isolated middle-aged neutron stars (see, e.g., Kaminker et al. 2002; Yakovlev and Pethick 2004). The chosen phenomenological density dependence of the critical proton temperature in the stellar core, $T_{\mathrm{cp}}(\rho)$, is shown in Fig. 1. In the outer core, this superfluidity is moderately strong. It extends beyond 
the threshold for the direct Urca process and can suppress this process in stars with masses that do not exceed greatly $M_{\mathrm{th}}$. Nevertheless, it disappears at a sufficiently high density (the repulsive component of the proton-proton interaction begins to hamper the Cooper pairing; see, e.g., Lombardo and Schulze 2001). In fact, it does not suppress the neutrino luminosity via the direct Urca process in stars with $M \sim M_{\max }$. The neutron superfluidity in the stellar core is assumed to be weak (in our calculations, it is disregarded altogether).

We will pay particular attention to the thermal conductivity of neutron star cores. In the adopted model of a nucleon stellar core, the thermal conductivity is determined mainly by neutrons, electrons, and muons, $\kappa=\kappa_{e \mu}+\kappa_{n}$. The thermal conductivity $\kappa_{e \mu}$ calculated without including the Landau damping via the exchange of transverse plasmons in collisions of electrons and muons with charged particles had long been used in the literature. In the absence of nucleon superfluidity, it dominated over the neutron thermal conductivity $\kappa_{n}\left(\kappa \approx \kappa_{e \mu}\right)$. However, as was shown by Heiselberg and Pethick (1993) (for a plasma of relativistic quarks), including the Landau damping can reduce appreciably the thermal conductivity. Shternin and Yakovlev (2007) reconsidered $\kappa_{e \mu}$ by taking into account the Landau damping and showed that (in the absence of superfluidity) the Landau damping reduces $\kappa_{e \mu}$ below $\kappa_{n}$, so that $\kappa \approx \kappa_{n}$.

In Fig. 2, $\kappa$ is plotted against density in the inner crust and core of a neutron star for two temperatures of matter, $T=10^{8}$ and $10^{9} \mathrm{~K}$. The thermal conductivity $\kappa_{n}$ was calculated using results from Baiko et al. (2001). The solid and dashed lines indicate the thermal conductivity in a nonsuperfluid core and in the presence of proton superfluidity, respectively. Superfluidity increases the thermal conductivity, because it suppresses the scattering of electrons, muons, and neutrons by protons and changes the plasma screening via charged particle scattering (Shternin and Yakovlev 2007). Curves $l$ indicate the thermal conductivity without including the Landau damping. We see that the new thermal conductivity is more than an order of magnitude lower than the old one. The Landau damping can also reduce appreciably the electron thermal conductivity in the crust of a cool neutron star (Shternin and Yakovlev 2006). This effect was included in our calculations, but it does not affect the cooling of young neutron stars.

In principle, heat can also be transferred in neutron stars by convection. Convection is known to play a crucial role in the evolution of a proto-neutron star ( $t \lesssim 1$ min; see, e.g., Imshennik and Litvinova 2006; Dessart et al. 2006; and references therein). However, in the case of interest to us (ordinary neutron stars; $t \gtrsim 1 \mathrm{yr}$ ), convection probably plays no particular

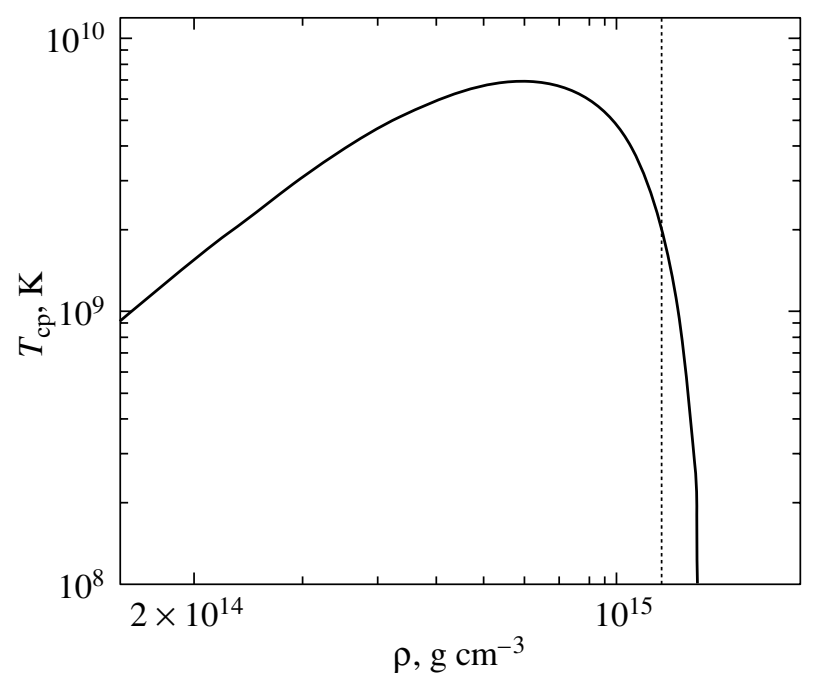

Fig. 1. Critical temperature for proton superfluidity in the stellar core versus density of matter. The vertical line indicates the direct Urca threshold.

role. The search for convective layers was included in neutron star cooling codes. Thin layers emerge near the surface of a neutron star (see, e.g., Miralles et al. 1997; Shibanov et al. 1998), but they do not affect its cooling.

In our calculations, we used the standard physics of a neutron star crust (Gnedin et al. 2001). The outer, heat-insulating stellar envelope is assumed to be composed of iron and to contain no magnetic field.

\section{THREE COOLING STAGES OF YOUNG NEUTRON STARS}

Figure 3 shows the profiles of temperature $T_{i}(\rho, t)=$ $T(\rho, t) \exp (\phi)$ inside a nonsuperfluid neutron star of mass $M=1.7 M_{\odot}\left(R=10.01 \mathrm{~km}, \rho_{\mathrm{c}}=2.700 \times 10^{15}\right.$ $\mathrm{g} \mathrm{cm}^{-3}$ ) at various times $t$ (for a remote observer), from the onset of cooling at $t=0$ to $50 \mathrm{yr}$. Here, $T$ is the true local temperature, while the factor $\exp (\phi)$ contains a metric function $\phi(\rho)$ and specifies the gravitational redshift (Shapiro and Teukolsky 1985). It is the temperature $T_{i}(\rho, t)$ that is constant throughout the star in thermal equilibrium, given the general relativity effects (Thorne 1977). At $t=0$, we set $T_{i}=10^{10} \mathrm{~K}$ in our calculations; this artificial initial condition is quickly "forgotten" and does not affect the subsequent evolution of the star.

According to Fig. 3, the inner core of a massive star where the direct Urca process is open cools faster than its outer core at $t \lesssim 20 \mathrm{yr}$. The temperature distribution turns out to be heterogeneous, with great temperature differences at the crust-core and innerouter core boundaries and in the thin heat-insulating envelope near the stellar surface. However, as the star 


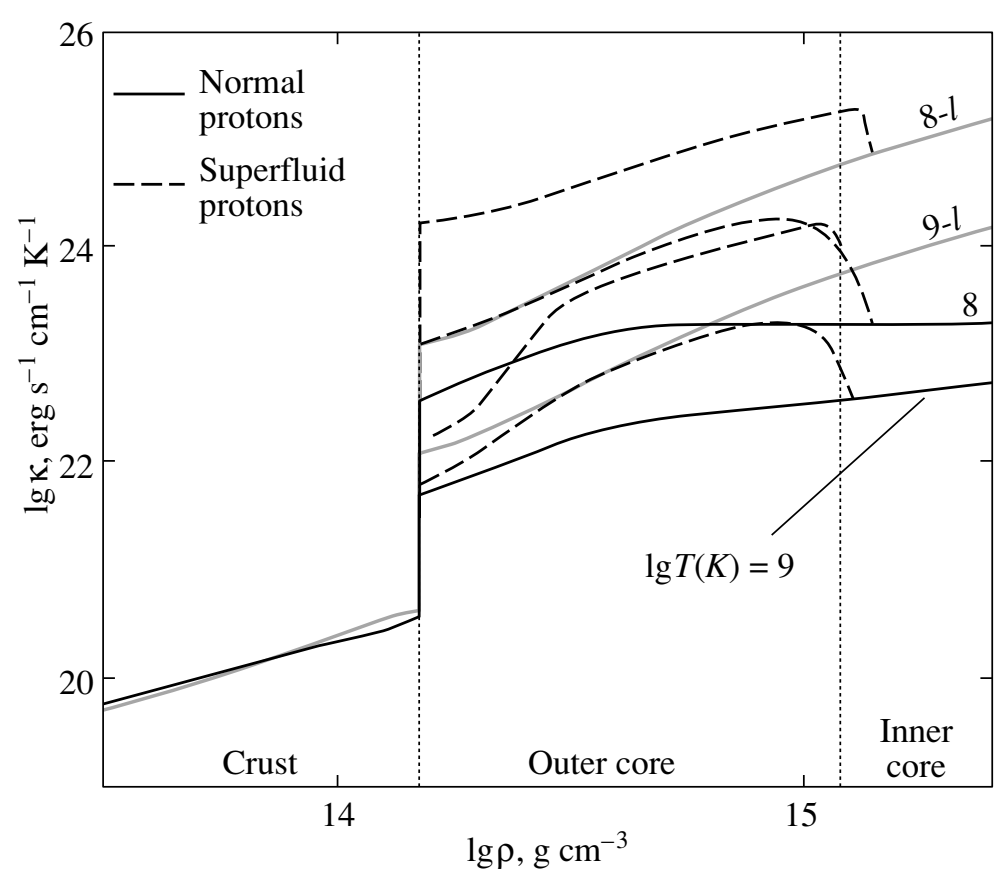

Fig. 2. Thermal conductivity versus density of matter in the inner crust and core of a neutron star for two temperatures, $T=10^{8}$ and $10^{9} \mathrm{~K}$. The solid and dashed lines correspond to nonsuperfluid matter and matter with superfluid protons in the stellar core, respectively. Curves $l$ indicate the thermal conductivity used previously (see the text).

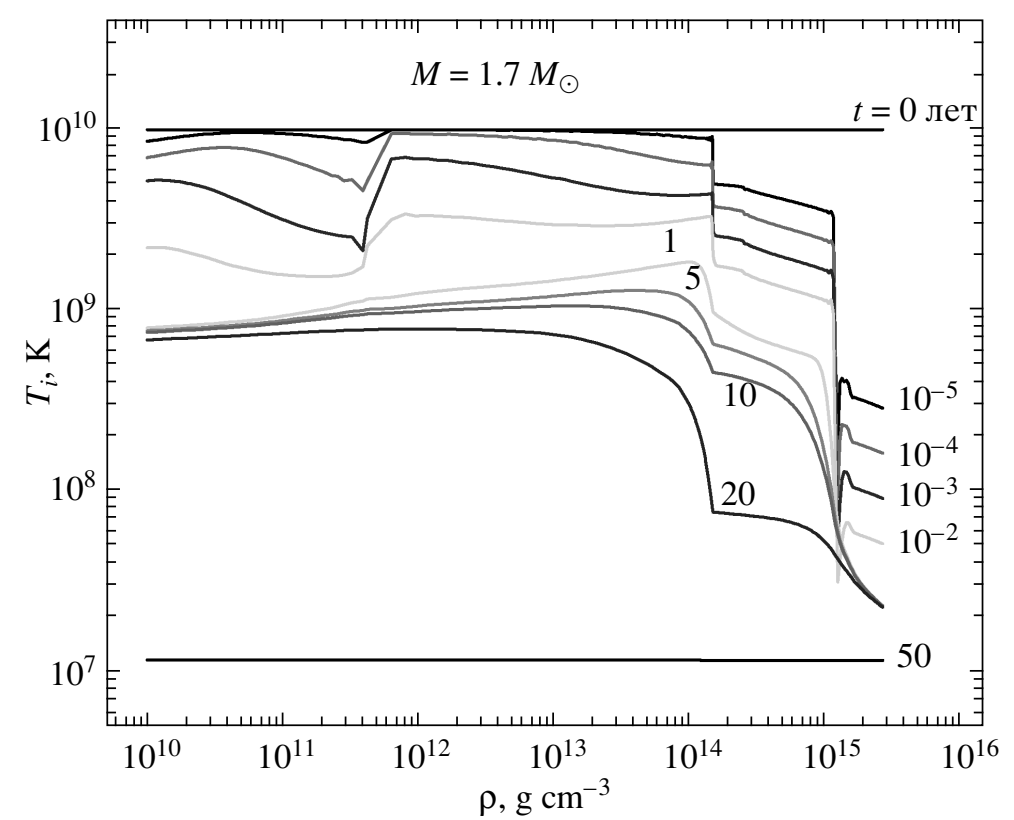

Fig. 3. Profiles of temperature $T_{i}(\rho)$ in a nonsuperfluid neutron star of mass $M=1.7 M_{\odot}$ at various times (from the top downward, $t=0,10^{-5}, 10^{-4}, 10^{-3}, 10^{-2}, 1,5,10,20$, and $50 \mathrm{yr}$ ).

cools, the neutrino emission weakens and the thermal conductivity equalizes the internal temperature. The temperature difference between the inner and outer cores is generally equalized faster than that between the core and the crust. The temperature equalization in rapidly cooling neutron stars was analyzed in detail by Lattimer et al. (1994). The temperature in lowmass stars (with the forbidden direct Urca process) is equalized appreciably longer (Gnedin et al. 2001).

Figure 4 shows the cooling curves $L_{\gamma}^{\infty}(t)$ for ASTRONOMY LETTERS Vol. 34 No. 102008 


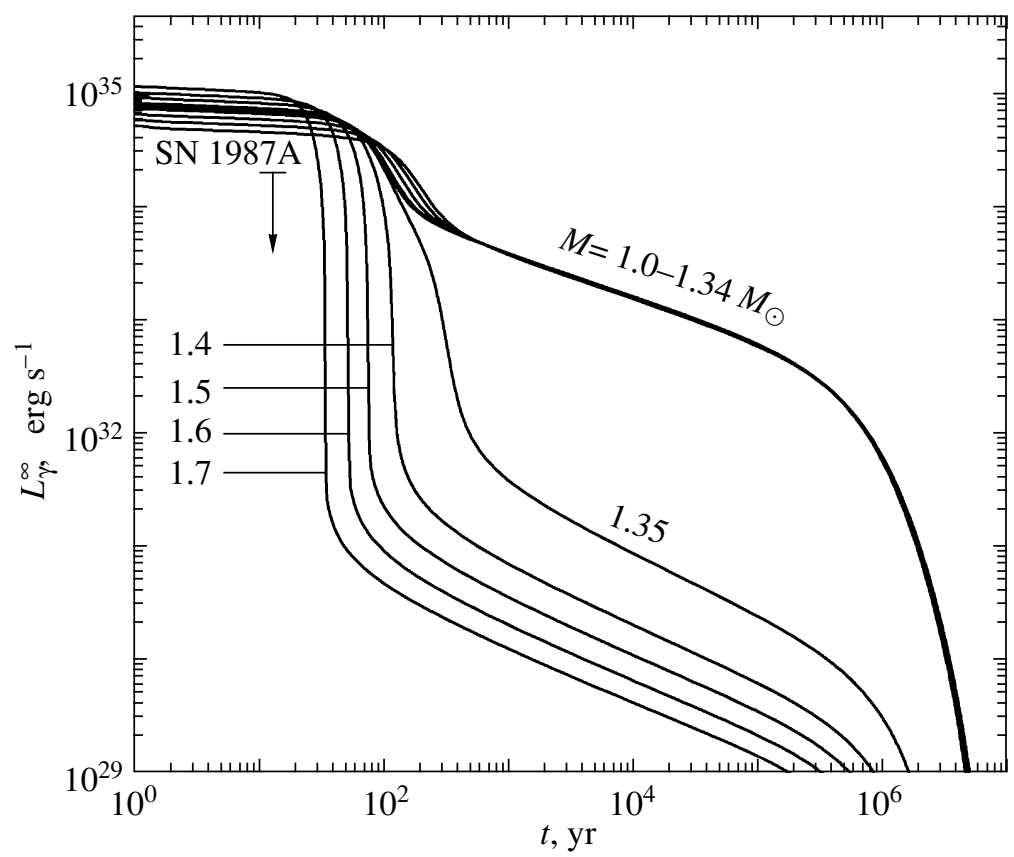

Fig. 4. Cooling curves for nonsuperfluid neutron stars of various masses. The vertical arrow indicates the upper limit (1) on the thermal luminosity of the hypothetical neutron star in the SN 1987A remnant (if the star is not hidden by a dense envelope).

nonsuperfluid neutron stars of various masses $(M=$ $1.0,1.1, \ldots, 1.7 M_{\odot}$ as well as 1.34 and $\left.1.35 M_{\odot}\right)$. The luminosity $L_{\gamma}^{\infty}(t)$ of a young neutron star evolves in three stages, with the changes in the internal thermal structure of the stars being much more varied than the changes in $L_{\gamma}^{\infty}(t)$.

The first stage corresponds to an early phase of internal thermal relaxation. It lasts until a noticeable drop in luminosity $L_{\gamma}^{\infty}(t)$ (the plateau of the cooling curves in Fig. 4). At this stage, the internal temperature distribution is highly nonuniform. The stellar crust is appreciably hotter than the core; the luminosity $L_{\gamma}^{\infty}(t)$ is insensitive to the physics of the inner stellar crust and core (in particular, to the opening of the direct Urca process) but is determined by the properties of the surface layers.

The second stage begins when the internal thermal relaxation ends. It is accompanied by a noticeable drop in luminosity $L_{\gamma}^{\infty}(t)$. It is convenient to introduce the relaxation time $t_{r}$ (Lattimer et al. 1994) as the time of the fastest decrease in $L_{\gamma}^{\infty}(t)$. The observation of thermal radiation from a neutron star at this stage can, in principle, allow the time $t_{r}$ to be measured. It is determined by the physical properties of the stellar layers with the most protracted relaxation. For the conditions in Fig. 4, these include the inner crust at the boundary with the core. The time $t_{r}$ is then determined by the heat capacity, thermal conductivity, and neutrino emission of the crust and by its thickness (Lattimer et al. 1994; Gnedin et al. 2001).
In addition, $t_{r}$ depends, though more weakly, on the conditions in the stellar core, primarily on whether the direct Urca process is present. In stars with $M \leq$ $M_{\text {th }} \approx 1.34 M_{\odot}$, this process is forbidden in Fig. 4; their relaxation time $t_{r} \sim 100-200 \mathrm{yr}$. In more massive stars, the direct Urca process is permitted and the relaxation is faster $\left(t_{r} \sim 30 \mathrm{yr}\right.$ at $\left.M \approx M_{\max }\right)$. The times $t_{r}$ satisfy simple similarity relations; for low- and high-mass stars, these relations are different (Lattimer et al. 1994; Gnedin et al. 2001).

Note that $t_{r}$ depends on the thermal conductivity of the stellar cores. As an example, Fig. 5 shows the cooling of nonsuperfluid neutron stars with several masses. The solid and dashed curves were calculated with the new and old (see Fig. 2) thermal conductivities, respectively. The decrease in thermal conductivity under the action of Landau damping increases the relaxation time but, generally, by no more than $20 \%$ (whereas any increase in thermal conductivity in the core would not change the relaxation time at all). However, for stars with a mass $M$ that is only slightly higher than the threshold mass $M_{\text {th }}$ (having a tiny inner core with a high neutrino luminosity), the relaxation is longer by a factor of several. Thus, for example, for a star with $M=1.35 M_{\odot}$, we have $t_{r} \approx 100 \mathrm{yr}$ for the old thermal conductivity and $t_{r} \approx 300 \mathrm{yr}$ for the new one in Fig. 5. Note also that the cooling of a star of any mass after its relaxation is excellently described in the approximation of isothermal inner layers and does not depend on the thermal conductivity in the core at all. 


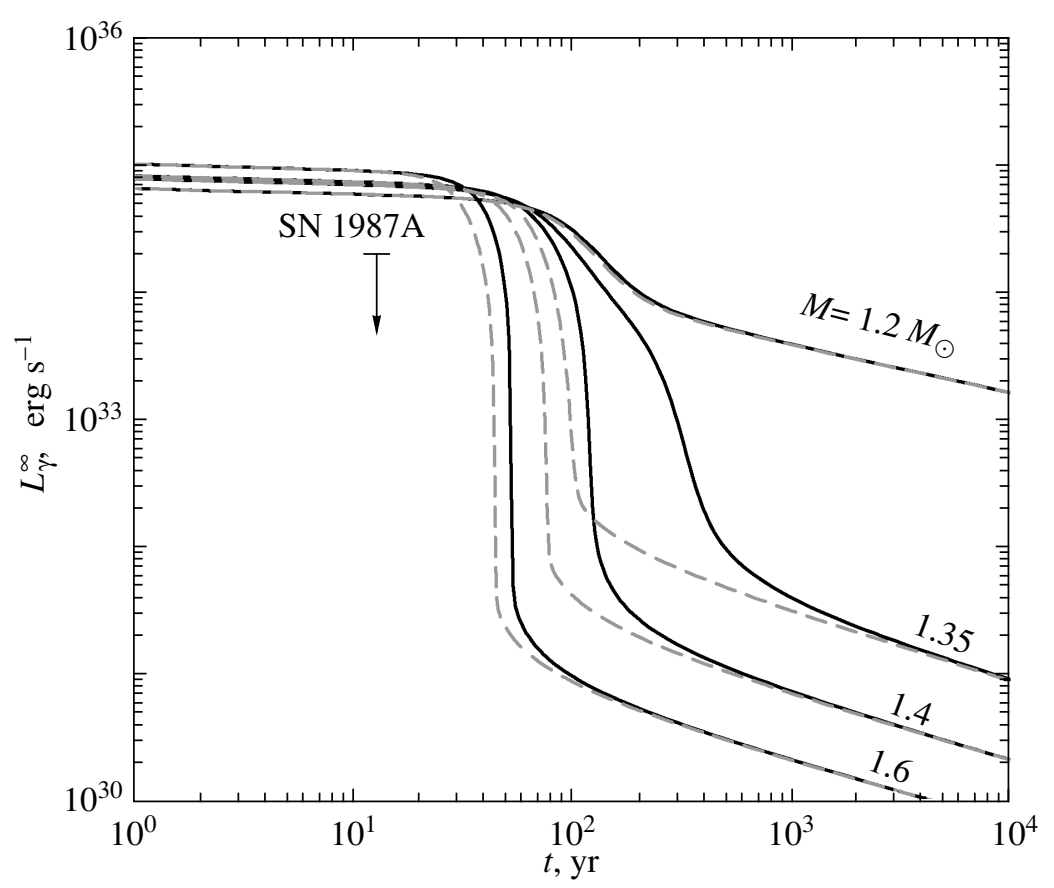

Fig. 5. Cooling curves for nonsuperfluid neutron stars of several masses calculated with the new (solid lines) and old (dashed lines) thermal conductivities in the stellar cores.

The third stage is the cooling of an internally equilibrium star after its relaxation $\left(t \gtrsim t_{r}\right)$. It is this cooling that is usually studied in the literature and is compared with observations. It is governed by the heat capacity and neutrino emission of the stellar core and by the thermal conductivity of the heatinsulating surface layers. For the conditions in Fig. 4, two distinctly different populations of neutron stars emerge at this stage: rapidly $\left(M<M_{\text {th }}\right)$ and slowly ( $M>M_{\text {th }}$ ) cooling stars separated by a very narrow range of masses, $\Delta M \lesssim 0.01 M_{\odot}$. These results are in conflict with the observations of isolated middle-aged neutron stars (see, e.g., Kaminker et al. 2002).

Figure 6 shows the cooling of neutron stars of various masses with proton superfluidity (see Fig. 1). Superfluidity does not change the stellar luminosity $L_{\gamma}^{\infty}(t)$ at the first cooling stage, but it becomes important at the subsequent stages. It reduces the neutrino luminosity of low-mass stars and slows down their cooling (Kaminker et al. 2002). In addition, it smears the direct Urca threshold and ensures a smooth transition from slow to rapid cooling. As a result, three representative types of neutron stars are formed: slowly cooling low-mass stars, rapidly cooling high-mass stars, and moderately rapidly cooling intermediate-mass stars. This explains the observations of cooling middle-aged neutron stars (Kaminker et al. 2002). As in the absence of superfluidity, the new thermal conductivities protract the relaxation (Fig. 7), though not so greatly.
Let us summarize the peculiarities of the cooling of young neutron stars.

(1) At the first cooling stage, the luminosity $L_{\gamma}^{\infty}$ is determined only by the properties of the stellar surface layers. The luminosity of a star with an age of $1 \mathrm{yr}$ $\lesssim t \lesssim t_{r}$ is approximately constant (the plateau on the cooling curves, Figs. $4-7$ ) and is

$$
L_{\gamma}^{\infty} \sim 10^{35}-10^{36} \mathrm{erg} \mathrm{s}^{-1},
$$

corresponding to a surface temperature $T_{\mathrm{s}}^{\infty} \sim(3-$ $6) \times 10^{6} \mathrm{~K}$. The lower values of $L_{\gamma}^{\infty}$ and $T_{\mathrm{s}}^{\infty}$ correspond to the presented calculations. The upper values are obtained if we assume that the star has an accretion envelope of light elements (with reduced heat insulation; Potekhin et al. 1997).

(2) After thermal relaxation $\left(t \sim t_{r}\right)$, the luminosity $L_{\gamma}^{\infty}(t)$ decreases appreciably. The relaxation time $t_{r}$ is determined mainly by the properties of the stellar crust and, to a lesser extent, by the properties of the core. Under ordinary assumptions about the crust and core properties (in Figs. 4-7),

$$
t_{r} \gtrsim 30-300 \mathrm{yr} \text {. }
$$

(3) The subsequent cooling of internally equilibrium stars $\left(t \gtrsim t_{r}\right)$ is governed by the physics of their core, their mass, and the properties of the outer envelopes, which has been well studied in the literature. 


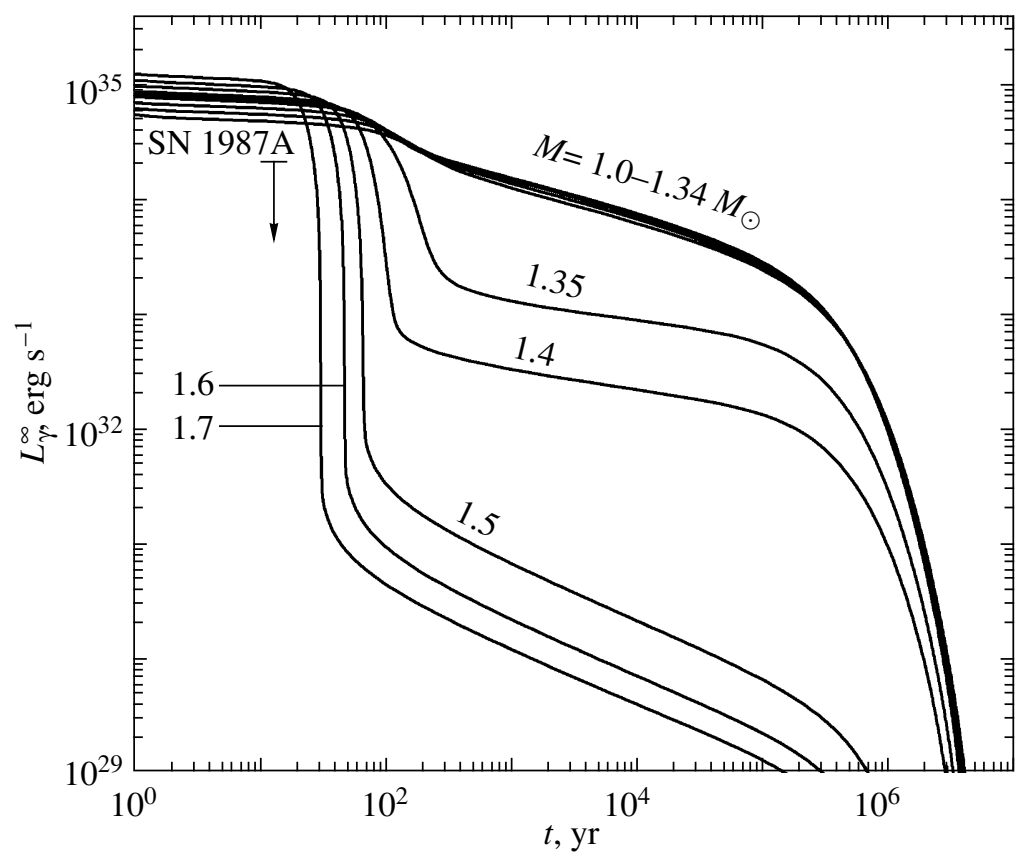

Fig. 6. Cooling of neutron stars of various masses with proton superfluidity in the stellar cores.

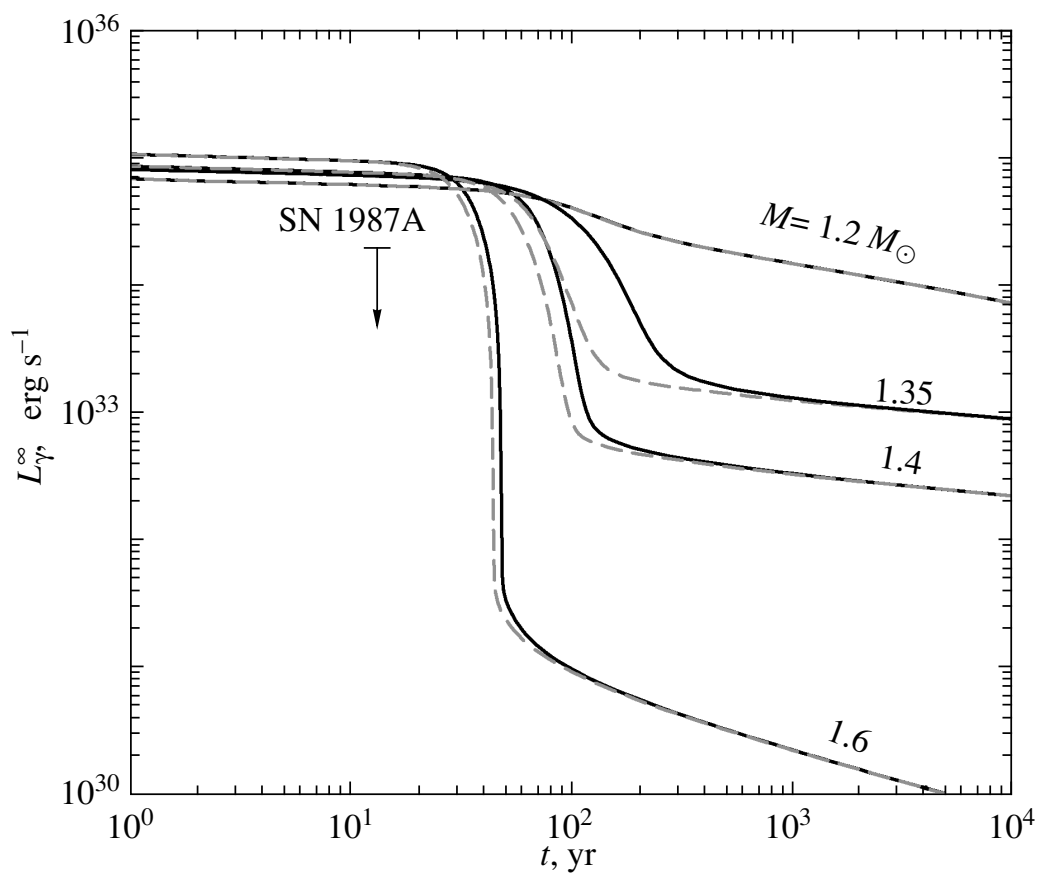

Fig. 7. Cooling of superfluid neutron stars of several masses calculated with the new (solid lines) and old (dashed lines) thermal conductivities.

\section{THEORY AND OBSERVATIONS}

Let us compare the cooling theory with the observational upper limit on the thermal luminosity (1) of the hypothetical neutron star in the SN 1987A remnant. Several cases are possible.

\section{A Neutron Star with a Normal Relaxation Time}

We have in mind the typical relaxation time (3) obtained in our calculations under ordinary assumptions about the properties of the stellar matter. In this case, the age $t \approx 13-14 \mathrm{yr}$ is shorter than the relaxation time (3) and a star of this age is at the first 


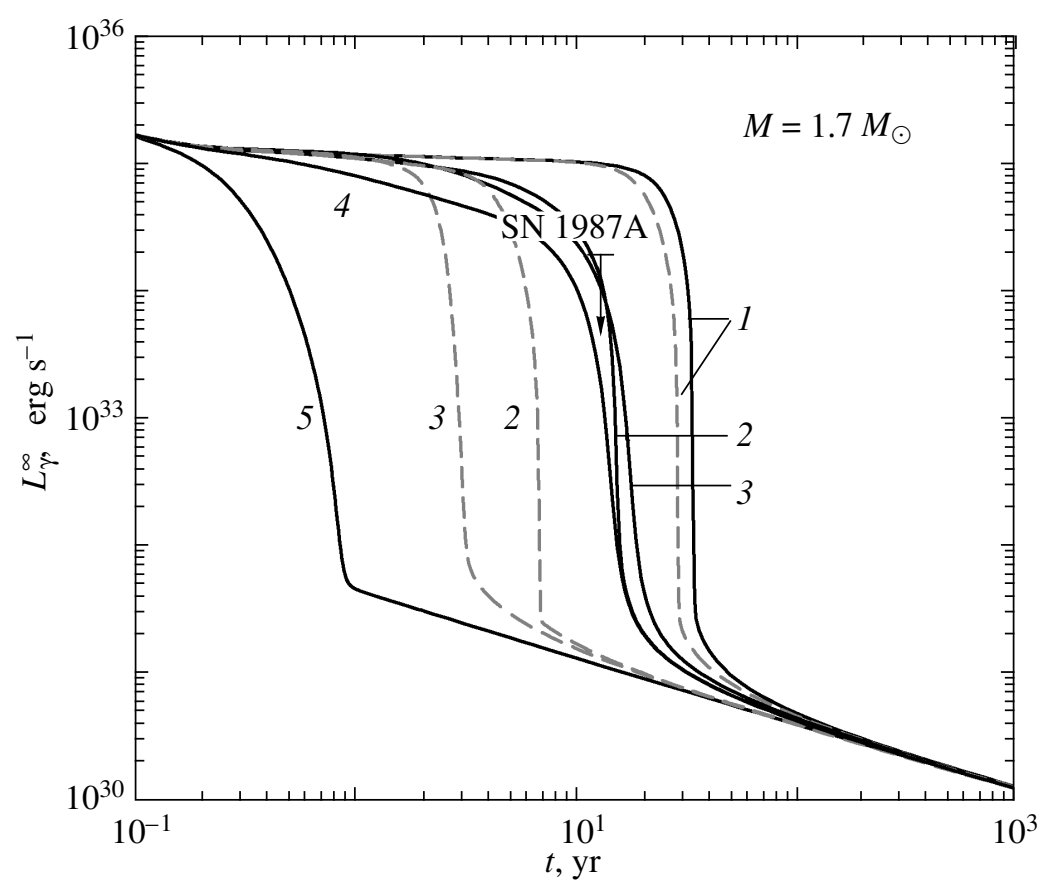

Fig. 8. Cooling of a neutron star with $M=1.7 M_{\odot}$ and a nonsuperfluid core: 1 , normal cooling; 2 , zero heat capacity of free neutrons in the inner crust; 3 , infinite thermal conductivity in the inner crust; 4 , both factors 2 and 3 were taken into account; 5 , infinite thermal conductivity in the inner crust and the core. The solid and dashed lines indicate the new and old thermal conductivities, respectively.

cooling stage. Its theoretical luminosity (2) exceeds the observational limit (1) by a factor of 20-100. Such a star should be hidden in the center of the $\mathrm{SN}$ remnant. For the minimum theoretical luminosity and a thermal spectrum, the optical depth at the maximum of the spectrum $(h \nu \sim 0.7 \mathrm{keV})$ should exceed $\tau(0.7 \mathrm{keV}) \gtrsim 3$ (and the total hydrogen column density to the star in the remnant at Galactic heavy-element abundances is $N_{\mathrm{H}} \gtrsim 10^{22} \mathrm{~cm}^{-2}$ ). For the maximum theoretical luminosity, we have, respectively, $\tau(1.4 \mathrm{keV}) \gtrsim 4.5\left(N_{\mathrm{H}} \gtrsim 5 \times 10^{22} \mathrm{~cm}^{-2}\right)$. These results are consistent with the optical depth of the SN remnant, $\tau(5 \mathrm{keV}) \sim 7$, estimated by Shtykovskiy et al. (2005).

If the aforesaid is true, then the completion of the star's thermal relaxation is yet to come. It will possibly be observable once the center of the SN 1987A remnant becomes transparent as a noticeable decrease in stellar luminosity $L_{\gamma}^{\infty}(t)$. If one succeeds in determining $t_{r}$, then it will be possible to impose constraints on the thickness of the envelope and on the physical properties of the inner stellar layers that determine $t_{r}$.

\section{A Neutron Star with a Short Relaxation Time}

The neutron star may not be hidden by an opaque envelope. The thermal relaxation then has been completed $\left(t>t_{r}\right)$ and the star has become cool (its luminosity dropped below limit (1)). The theory allows this possibility under the following assumptions. First, the neutron star should be massive enough. Its neutrino luminosity should be enhanced (e.g., by the direct Urca process unsuppressed by superfluidity at the stellar center). Otherwise, the relaxation process would be protracted and indistinct. Second, to shorten the relaxation, the star should possess a thin crust. Subsequently, short relaxation is achieved by the suppression of the heat capacity of free neutrons in the inner stellar crust by strong neutron superfluidity (with a critical temperature $T_{\mathrm{cn}}(\rho) \gtrsim 3 \times 10^{9} \mathrm{~K}$ ).

Finally, short relaxation is possible at an anomalously high thermal conductivity of the crust. This assumption is unusual for cooling calculations, but it is allowed by the theory. It may well be that strong neutron superfluidity in the inner crust gives rise to a very efficient mechanism of heat transfer by convective motions of the normal neutron liquid component in the presence of a temperature gradient. This mechanism is well known from laboratory experiments with liquid ${ }^{4} \mathrm{He}$ (see, e.g., D. Tilley and J. Tilley 1990). No temperature gradient can be produced in pure ${ }^{4} \mathrm{He}$ under experimental conditions - it is immediately smeared by the emerging convective motions. A similar mechanism can also operate in the inner crust of a neutron star and can make it isothermal. It was mentioned in astrophysical literature (Flowers 


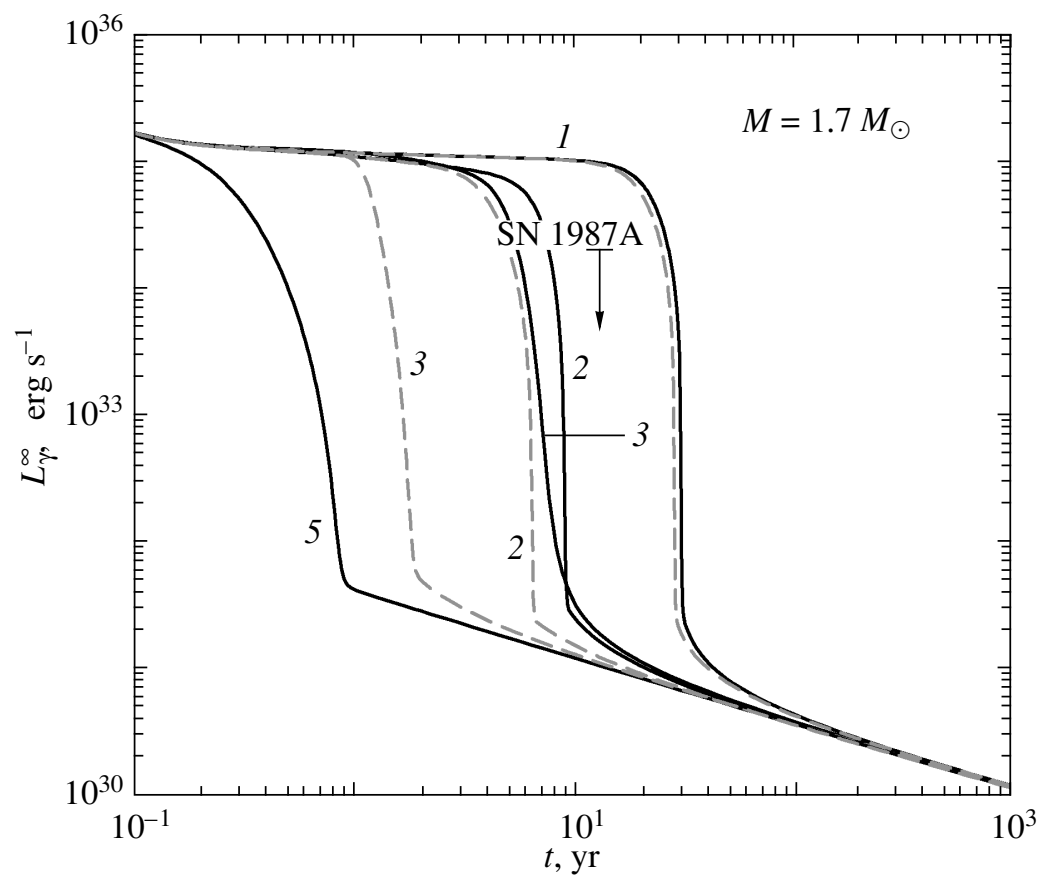

Fig. 9. Same as Fig. 8 but with proton superfluidity in the stellar core. Curve 4 is not shown in order not to overload the figure.

and Itoh 1976, 1979), but it has not been developed quantitatively.

The aforesaid is illustrated in Figs. 8 and 9, which show the cooling curves for a massive $\left(M=1.7 M_{\odot}\right)$ neutron star without and with proton superfluidity in its core. Curves 2 correspond to extremely strong superfluidity of free neutrons in the inner core, which completely suppresses the neutron heat capacity. Curves 3 were calculated by assuming that the thermal conductivity of the inner crust was infinite. Curve 4 in Fig. 8 corresponds to an infinite thermal conductivity and a zero heat capacity of free neutrons in the inner crust. We see that proton superfluidity in the stellar core accelerates noticeably the relaxation. In contrast, using the new thermal conductivity in the core for unusual properties of the crust decelerates greatly the relaxation. The thermal conductivity of the core is particularly important in the model of an isothermal crust (since the main relaxation in this model takes place not in the stellar crust but in the core). As a result, curve 4 in Fig. 8, along with curves 2-3 in Fig. 9, calculated with the new thermal conductivity actually give the required short relaxation time $\left(t_{r}<t\right)$. Even shorter relaxation is achieved by assuming the inner crust and the core to be isothermal (curves 5). In this case, relaxation takes place only in the thin outer crust.

Short relaxation is also characteristic of strange stars with a crust. Strange stars composed mainly of quark matter can have only a thin crust of normal matter that extends to a density no higher than the neutronization density in the crust of an ordinary neutron star $\left(\rho \lesssim 4 \times 10^{11} \mathrm{~g} \mathrm{~cm}^{-3}\right)$. The thermal relaxation in a strange star with a crust is determined by the diffusion of heat through the thin crust and can take only a few hours (see, e.g., Page et al. 2006).

Thus, the cooling theory allows the thermal relaxation time to be shortened to $t_{r} \lesssim t$, consistent with the assumption that the star is not obscured by an expanding shell.

\section{Other Scenarios}

Of course, other scenarios that explain the nondetection of a neutron star in the SN 1987A remnant are also possible.

In particular, we can combine the two cases described above and assume that the star is slightly obscured by the center of the SN shell and has a shortened relaxation time (e.g., solid curves 2 and 3 in Fig. 8).

Another possibility is to take a faint upper limit on the luminosity of the point object in the SN 1987A remnant (Shtykovskiy et al. 2005; see above) instead of (1). It is higher than the theoretically predicted limit (2). In this case, any cooling neutron star (hidden or unhidden by the SN shell) is so far unobservable.

Finally, it may well be that the neutron star in the SN 1987A remnant was formed and then collapsed into a black hole, for example, after the deceleration of a very rapid rotation or as a result of accretion 
and subsequent loss of stability (see, e.g., Fryer et al. 1999; Imshennik and Ryazhskaya 2004; and references therein).

\section{CONCLUSIONS}

We have shown that the observations of the SN 1987A remnant and the cooling theory for young neutron stars are consistent with the fact that a neutron star was formed in the SN 1987A remnant. There are the two most likely possibilities.

(1) The neutron star has the normal thermal relaxation time (3), but it is hidden in the opaque center of the SN shell. The shell should then absorb the thermal X-ray radiation from the star by more than a factor of 5 , while the (unobservable) star has not yet reached thermal relaxation. When the star is detected once the shell has become transparent, there is a chance of observing a decrease in the thermal luminosity of the star after its thermal relaxation and imposing constraints on the properties of the matter in the inner stellar layers.

(2) The star is not hidden by the SN shell, but it has cooled; the thermal relaxation in it has been completed. Such a short relaxation time (less than $14 \mathrm{yr}$ ) is possible only if the star undergoes intense neutrino cooling and has a thin crust with strong neutron superfluidity and/or an anomalously high thermal conductivity. Short relaxation times are also typical of strange stars with a crust.

We also showed that the relaxation time depends on the thermal conductivity of the superdense stellar core, particularly in the scenarios with a short relaxation time.

In any case, the searches for a young neutron star in the SN 1987A remnant should be continued.

\section{ACKNOWLEDGMENTS}

We wish to thank S.A. Grebenev, A.M. Krasilshchikov, S.B. Popov, and Yu.A. Shibanov for the discussions and the referee for the critical remarks. This work was supported by the Dynasty Foundation, the Russian Foundation for Basic Research (project nos. 08-02-00837 and 05-02-22003), and the Presidential Program for State Support of Leading Scientific Schools (project no. NSh 2600.2008.2).

\section{REFERENCES}

1. D. Arnett, Supernovae and Nucleosynthesis: An Investigation of the History of Matter, from the Big Bang to the Present (Princeton: Princeton Univ. Press, 1996).

2. D. A. Baiko, P. Haensel, and D. G. Yakovlev, Astron Astrophys. 374, 151 (2001)
3. M. Bejger, D. G. Yakovlev, and O. Y. Gnedin, Acta Physica Polonica B 34, 223 (2003).

4. D. N. Burrows, E. Michael, U. Hwang, et al., Astrophys. J. 543, L149 (2000).

5. L. Dessart, A. Burrows, E. Livne, and C. D. Ott, Astrophys. J. 645, 534 (2006).

6. E. Flowers and N. Itoh, Astrophys. J. 206, 218 (1976).

7. E. Flowers and N. Itoh, Astrophys. J. 230, 847 (1979).

8. C. Fransson and R. A. Chevalier, Astrophys. J. 322, L15 (1987).

9. C. L. Fryer, S. A. Colgate, and P. A. Pinto, Astrophys. J. 511, 885 (1999).

10. U. Geppert, D. Page, and T. Zannias, Astron. Astrophys. 345, 847 (1999).

11. O. Y. Gnedin, D. G. Yakovlev, and A. Y. Potekhin, Mon. Not. R. Astron. Soc. 324, 725 (2001).

12. G. J. M. Graves, P. M. Challis, R. A. Chevalier, et al., Astrophys. J. 629, 944 (2005).

13. F. Haberl, U. Geppert, B. Aschenbach, and G. Hasinger, Astron. Astrophys. 460, 811 (2006).

14. P. Haensel, A. Y. Potekhin, and D. G. Yakovlev, Neutron Stars. 1. Equation of State and Structure (N.Y.: Springer, 2007).

15. H. Heiselberg and C. J. Pethick, Phys. Rev. D 48, 2916 (1993).

16. S. Immler, K. Weiler, and R. McCray, eds., Supernova 1987A: 20 Years After: Supernovae and Gamma-Ray Bursters, AIP Conf. Proc. 937 (2007).

17. V. S. Imshennik and D. K. Nadyozhin, Usp. Fiz. Nauk 156, 561 (1988).

18. V. S. Imshennik and O. G. Ryazhskaya, Pis'ma Astron. Zh. 30, 17 (2004) [Astron. Lett. 30, 14 (2004)].

19. V. S. Imshennik and I. Yu. Litvinova, Yad. Fiz. 69, 660 (2006) [Phys. At. Nucl. 69, 636 (2006)].

20. A. D. Kaminker, D. G. Yakovlev, and O. Y. Gnedin, Astron. Astrophys. 383, 1076 (2002).

21. J. M. Lattimer, C. J. Pethick, M. Prakash, and P. Haensel, Phys. Rev. Lett. 66, 2701 (1991).

22. J. M. Lattimer, K. A. van Riper, M. Prakash, and M. Prakash, Astrophys. J. 425, 802 (1994).

23. R. N. Manchester, AIP Conf. Proc. 937, 134 (2007).

24. J. A. Miralles, V. Urpin, and K. Van Riper, Astrophys. J. 480, 358 (1997)

25. A. Muslimov and D. Page, Astrophys. J. 440, 77 (1995).

26. K. Nomoto and S. Tsuruta, Astrophys. J. 312, 711 (1987).

27. D. Page, U. Geppert, and F. Weber, Nucl. Phys. A 777, 497 (2006).

28. D. Page and J. H. Applegate, Astrophys. J. 394, L17 (1992).

29. S. Park, S. A. Zhekov, D. N. Burrows, et al., Astrophys. J. 610, 275 (2004).

30. J. W. Percival, P. T. Boyd, J. D. Biggs, et al., Astrophys. J. 446, 832 (1995).

31. A. Y. Potekhin, G. Chabrier, and D. G. Yakovlev, Astron. Astrophys. 323, 415 (1997).

32. M. Prakash, T. L. Ainsworth, and J. M. Lattimer, Phys. Rev. Lett. 61, 2518 (1988).

ASTRONOMY LETTERS Vol. 34 No. 102008 
33. S. Shapiro and S. Teukolsky, Black Holes, White Dwarfs and Neutron Stars: The Physics of Compact Objects (Wiley, New York, 1983; Mir, Moscow, 1985), vols. 1, 2.

34. Yu. A. Shibanov, A. Y. Potekhin, D. G. Yakovlev, and V. E. Zavlin, The Many Faces of Neutron Stars (Dordrecht: Kluwer, 1998), p. 553.

35. P. S. Shternin and D. G. Yakovlev, Phys. Rev. D 74, 043004 (2006).

36. P. S. Shternin and D. G. Yakovlev, Phys. Rev. D 75, 103004 (2007).

37. P. E. Shtykovskiy, A. A. Lutovinov, M. R. Gilfanov, and R. A. Sunyaev, Pis'ma Astron. Zh. 31, 258 (2005) [Astron. Lett. 31, 258 (2005)].
38. K. S. Thorne, Astrophys. J. 212, 825 (1977).

39. D. R. Tilley and J. Tilley, Superfluidity and Superconductivity (Bristol: IOP Publ., 1990).

40. S. E. Woosley and T. A. Weaver, Astrophys. J. Suppl. Ser. 101, 181 (1995).

41. D. G. Yakovlev, A. D. Kaminker, O. Y. Gnedin, and P. Haensel, Phys. Rep. 354, 1 (2001).

42. D. G. Yakovlev and C. J. Pethick, Annu. Rev. Astron. Astrophys. 42, 169 (2004).

Translated by V. Astakhov 